\title{
Effect of tryptophan supplementation on diet-induced non-alcoholic fatty liver disease in mice
}

\author{
Yvonne Ritze, Gyöngyi Bárdos, Astrid Hubert, Maureen Böhle and Stephan C. Bischoff* \\ Department of Nutritional Medicine, University of Hohenheim, Fruwirthstrasse 12, Stuttgart 70599, Germany
}

(Submitted 23 May 2013 - Final revision received 25 November 2013 - Accepted 23 January 2014 - First published online 8 April 2014)

\section{Abstract}

Intestinal serotonin (5-hydroxytrypamine, 5-HT) metabolism is thought to play a role in gut functions by regulating motility, permeability and other functions of the intestine. In the present study, we investigated the effect of tryptophan (TRP), the precursor of 5-HT, supplementation on intestinal barrier functions and non-alcoholic fatty liver disease (NAFLD). An established mouse model of NAFLD induced by feeding a fructose-rich diet ( $\mathrm{N}$ group) was used in the present study. TRP was administered orally for 8 weeks to $\mathrm{C} 57 \mathrm{BL} / 6 \mathrm{~J}$ control or NAFLD mice. NAFLD-related liver parameters (hepatic TAG and Oil Red O staining), intestinal barrier parameters (tight-junction protein occludin and portal plasma lipopolysaccharides (LPS)) and 5-HT-related parameters (5-HT, 5-HT transporter (SERT) and motility) were measured. We observed reduced duodenal occludin protein concentrations $(P=0.0007)$, high portal plasma LPS concentrations $(P=0.005)$ and an elevated liver weight:body weight ratio $(P=0.01)$ in the $\mathrm{N}$ group compared with the parameters in the control group. TRP supplementation led to an increase in occludin concentrations $(P=0.0009)$ and consecutively reduced liver weight:body weight ratio $(P=0.009)$ as well as overall hepatic fat accumulation in the $\mathrm{N}$ group $(P=0.05)$. In addition, the $\mathrm{N}$ group exhibited reduced SERT protein expression $(P=0 \cdot 002)$, which was normalised by TRP supplementation $(P=0 \cdot 02)$. For the first time, our data indicate that oral TRP supplementation attenuates experimental NAFLD in mice. The underlying mechanisms are not clear, but probably involve stabilisation of the intestinal barrier in the upper small intestine and amelioration of the dysregulated intestinal serotonergic system.

Key words: Tryptophan: Non-alcoholic fatty liver disease: Intestinal permeability: Serotonin: Serotonin re-uptake transporter

The prevalence of non-alcoholic fatty liver disease (NAFLD) is increasing worldwide, with up to $33 \%$ of adults being affected in Western countries ${ }^{(1)}$. This alarming increase in the number of individuals affected is related to the contemporary pandemic of obesity fostered by an unhealthy dietary pattern and a sedentary lifestyle, consequently leading to an increase in the risk of death $^{(2)}$. NAFLD patients exhibit signs of liver inflammation and increased hepatic lipid accumulation ${ }^{(3)}$. In addition, the development of NAFLD in obese individuals is closely associated with insulin resistance and other metabolic disorders and thus might be of clinical relevance ${ }^{(4)}$. We and others demonstrated that not only overfeeding but also particular nutrients such as fructose and SFA promote NAFLD in both animals and humans ${ }^{(5-10)}$. Our findings might explain, at least in part, why many, but by far not all, obese individuals develop the metabolic syndrome and associated diseases. In addition, we provided evidence for the involvement of the intestinal serotonergic system in the development of NAFLD and obesity ${ }^{(7,11,12)}$.

Intestinal serotonin (5-hydroxytryptamine, 5-HT) is released by enterochromaffin cells and neurons and is regulated via the serotonin re-uptake transporter (SERT) ${ }^{(13)}$. The SERT is located on epithelial cells and neurons in the intestine ${ }^{(13)}$. Previously, we had tested the hypothesis that the dysregulation of 5-HT due to a diet-induced reduction or a genetically induced loss of the SERT is involved in monosaccharide-induced NAFLD $^{(7)}$. We clearly demonstrated that impairment of SERT promotes the fructose- and glucose-induced damage of the intestinal barrier leading to lipopolysaccharide (LPS) translocation and liver inflammation causing NAFLD ${ }^{(7)}$. Our findings are consistent with those of studies showing that 5-HT is a key regulator not only of intestinal motility ${ }^{(14)}$ but also of inflammation $^{(15,16)}$ and intestinal permeability ${ }^{(17-19)}$. 5-HT is synthesised from the amino acid tryptophan (TRP). Therefore, TRP supplementation is thought to enhance 5-HT synthesis; however, the substrate seems to be a minor regulator of synthesis compared with the rate-determining enzymes tryptophan hydroxylase (TPH) 1 and TPH $2^{(20)}$. Nevertheless, in earlier studies, TRP supplementation has been shown to result in elevated small-intestinal 5-HT concentrations as well as in increased duodenal and ileal motility ${ }^{(21,22)}$. Concerning the

\footnotetext{
Abbreviations: 5-HT, serotonin; C group, control diet; C + TRP group, control diet supplemented with tryptophan; LPS, lipopolysaccharide; MZ, mouse breeding; NAFLD, non-alcoholic fatty liver disease; N group, NAFLD mice fed a fructose-rich diet; N + TRP group, NAFLD mice fed a fructose-rich diet supplemented with tryptophan; SERT, serotonin re-uptake transporter; TPH, tryptophan hydroxylase; TRP, tryptophan.
}

*Corresponding author: Professor Dr S. C. Bischoff, fax + 49711459 24343, email bischoff.stephan@uni-hohenheim.de 
development of NAFLD, studies in laying hens have suggested that TRP supplementation reduces hepatic lipid accumulation $^{(23,24)}$. Interestingly, another study has revealed that, on the one hand, 5-HT supports liver regeneration after partial hepatectomy and, on the other hand, promotes steatohepatitis ${ }^{(25)}$. Until today, studies concerning TRP supplementation are patchy and confusing. Thus, in the present study, we aimed to investigate the effect of TRP supplementation on NAFLD. In particular, we were interested in determining whether TRP supplementation affects the translocation of LPS from the intestine to the liver and the subsequent development of NAFLD. The present results indicate TRP supplementation to be a new approach for attenuating dietinduced fatty liver disease.

\section{Materials and methods}

\section{Animals and treatments}

Mice were housed in a pathogen-free barrier facility accredited by the Association for Assessment and Accreditation of Laboratory Animal Care. All procedures were approved by the local Institutional Animal Care and Use Committee (Regional Council Stuttgart). C57BL/6J mice aged 6-8 weeks were obtained from Janvier (Le-Genes-St-isle, France). The animals were divided into four groups of five to six mice and were fed different diets ad libitum over an 8-week period: (a) control diet (C group) (water and mouse breeding (MZ) diet; sniff ${ }^{\circledR}$, containing: $6 \%$ fat, $23 \%$ protein, $5 \cdot 2 \%$ sugars, vitamins and minerals); (b) NAFLD-inducing fructoserich diet ( $\mathrm{N}$ group) (30\% fructose solution and enriched MZ diet containing $7 \cdot 7 \%$ fat, $27 \cdot 6 \%$ protein, $8 \cdot 2 \%$ sugars and adjusted vitamins and minerals); (c) control diet supplemented with TRP ( $\mathrm{C}+$ TRP group) $(0.4 \mathrm{~g} / \mathrm{kg}$ body weight corresponding to $0.24 \mathrm{~g} / 100 \mathrm{~g}$ diet); (d) fructose-rich diet supplemented with TRP ( + TRP group). The two animal groups fed the fructose-rich diets consumed about 50\% less food (see Table 1), as has been reported earlier ${ }^{(5)}$, and were therefore fed a more energy-dense MZ diet to compensate partially for the reduced food intake. The amount of TRP administered was selected according to the method of Rogers \& Pesti ${ }^{(24)}$, who demonstrated reduced hepatic lipid accumulation in laying hens after TRP supplementation. Liquid and food intakes, as well as body weight, were recorded weekly. After 8 weeks of feeding, the mice were anaesthetised with $80 \mathrm{mg} / \mathrm{kg}$ body weight of ketamine and $6 \mathrm{mg} / \mathrm{kg}$ body weight of xylazine, both administered intraperitoneally; blood was collected from the portal vein just after anaesthetisation. After killing the mice, tissue samples of the small intestine and liver were collected and frozen immediately in liquid $\mathrm{N}_{2}$ or fixed in paraformaldehyde and frozen in OCT Tissue-Tek (Sakura Finetek) for further investigation.

\section{Determination of intestinal motility}

A charcoal solution $(0.5 \mathrm{~g}$ charcoal, $0.25 \mathrm{~g}$ gum Arabic and $5 \mathrm{ml} \mathrm{NaCl}(0.9 \%))$ was orally administered to the mice at a concentration of $15 \mu \mathrm{l} / \mathrm{kg}$ body weight $10 \mathrm{~min}$ before killing to determine small-intestinal motility. The migration of the charcoal solution from the pylorus to the small intestine was measured once the intestine was removed and gently, without tension, stretched.

\section{Determination of hepatic TAG concentrations}

Liver tissue samples weighing 50-100 mg were homogenised in ice-cold double-concentrated PBS. Tissue lipids were extracted with methanol $(100 \mu \mathrm{l})$-chloroform $(200 \mathrm{ml})$, dried and resuspended in 5\% fat-free bovine serum albumin. Colorimetric assessment of TAG concentrations was carried out using a commercially available kit (Randox). Values were normalised to protein concentrations determined using the Bradford assay in the liver homogenates (Bio-Rad Laboratories).

\section{Oil Red $O$ and haematoxylin and eosin staining of liver tissue sections}

To determine hepatic lipid accumulation, paraformaldehydefixed liver tissue sections $(10 \mu \mathrm{m})$ were stained with Oil

Table 1. Effect of tryptophan supplementation on physiological parameters§ (Mean values with their standard errors; $n 5-6$ )

\begin{tabular}{|c|c|c|c|c|c|c|c|c|}
\hline & \multicolumn{2}{|c|}{ C } & \multicolumn{2}{|c|}{$\mathrm{C}+\mathrm{TRP}$} & \multicolumn{2}{|c|}{$\mathrm{N}$} & \multicolumn{2}{|c|}{$\mathrm{N}+\mathrm{TRP}$} \\
\hline & Mean & SE & Mean & SE & Mean & SE & Mean & SE \\
\hline Liquid intake (ml/week) & $46 \cdot 1$ & 0.8 & $47 \cdot 2$ & 0.9 & $43 \cdot 0$ & 1.9 & $41 \cdot 7$ & $2 \cdot 3$ \\
\hline Food intake (g/week) & $24 \cdot 2$ & $1 \cdot 2$ & $28 \cdot 6$ & 0.4 & $11 \cdot 8 \dagger$ & 0.3 & $12 \cdot 6 \dagger$ & 0.7 \\
\hline Total energy intake (kJ/week) & 368 & 6 & $406^{\star}$ & 2 & 390 & 8 & 394 & 8 \\
\hline Weight gain $(\mathrm{g})$ & $5 \cdot 4$ & 0.6 & 5.4 & 0.3 & 5.5 & 0.5 & $5 \cdot 2$ & 0.4 \\
\hline Liver weight:body weight ratio (\%) & $5 \cdot 7$ & $0 \cdot 1$ & $5 \cdot 8$ & $0 \cdot 2$ & $6 \cdot 9^{*}$ & 0.3 & $5 \cdot 2 \ddagger$ & 0.4 \\
\hline Motility $(\mathrm{cm})$ & 11.4 & $2 \cdot 0$ & $16 \cdot 8$ & 1.8 & 13.9 & 1.9 & $17 \cdot 0$ & $2 \cdot 0$ \\
\hline Serotonin content $(\mathrm{ng} / \mathrm{mg})$ & $20 \cdot 8$ & $2 \cdot 9$ & $25 \cdot 6$ & $7 \cdot 6$ & 24.9 & $3 \cdot 3$ & $27 \cdot 2$ & $7 \cdot 6$ \\
\hline
\end{tabular}

$\mathrm{C}$, control diet group; C + TRP, control diet group with tryptophan supplementation; $\mathrm{N}$, non-alcoholic fatty liver disease mice fed a fructose-rich diet; N + TRP, non-alcoholic fatty liver disease mice fed a fructose-rich diet with tryptophan supplementation.

* Mean values were significantly different from those of the control group $(P<0.05)$.

† Mean values were significantly different from those of the $C+$ TRP group $(P<0.05)$.

$\ddagger$ Mean value was significantly different from that of the $\mathrm{N}+$ TRP group $(P<0.05)$.

$\S$ The detailed feeding protocols of the four animal groups are described in the Materials and methods section. 
differences between the groups, we used as a post hoc test an unpaired, two-tailed $t$ test or $t$ test with Welch's correction (if variances differed significantly), as we were interested in comparing the $\mathrm{C}$ and $\mathrm{N}$ groups as well as the $\mathrm{N}$ and $\mathrm{N}+$ TRP groups. A $P$ value $<0.05$ was determined as the level of significance before the start of the study. For calculation and graph design, the software GraphPad Prism 5 (GraphPad Software) was used.

\section{Results}

\section{Effect of tryptophan supplementation on physiological parameters}

Liquid intake and weight gain were similar in the four dietary groups (Table 1). Independent of TRP supplementation, we observed, as has been shown previously ${ }^{(5)}$, a reduction in solid food intake by $48.5-51.0 \%$ in mice given drinkingwater containing fructose (fructose-rich diet) compared with the food intake in mice given plain drinking-water.

\section{Effect of tryptophan supplementation on hepatic lipid accumulation}

The liver weight:body weight ratio $(P=0 \cdot 014)$ was significantly increased in the $\mathrm{N}$ group (Fig. 1(a)). TRP supplementation reduced the liver weight:body weight ratio to normal levels $\left(P=0.009\right.$; Fig. 1(a)). As has been shown previously ${ }^{(5)}$, here fructose-rich diet-fed mice exhibited a dramatic increase in overall hepatic lipid accumulation compared with the fructose-rich diet-fed mice in which hepatic lipid accumulation was reduced by TRP supplementation (Fig. 1(b) and (d)). Similarly, fructose-rich diet-fed mice exhibited a higher degree of steatosis, cellular ballooning and lobular inflammation compared with mice fed the fructose-rich diet with TRP supplementation (Fig. 1(e); Table 2). The NAFLD activity score was 3 for the $\mathrm{N}$ group and 0 for all the other groups (Table 2). Nevertheless, hepatic TAG concentrations were not significantly reduced in the TRP-supplemented $\mathrm{N}$ group (Fig. 1(c)).

\section{Effect of tryptophan supplementation on tight-junction protein expression}

We measured the expression of occludin, a tight-junction protein thought to be important for the epithelial barrier function in the small intestine ${ }^{(27)}$. Measurements in the duodenum, the first site of sugar absorption, revealed that occludin protein expression in the $\mathrm{N}$ group was significantly reduced $(P=0.0007)$ compared with that in the control group (Fig. 2(a)). Interestingly, TRP supplementation normalised duodenal occludin protein expression in the $\mathrm{N}$ group $(P=0.0009$; Fig. 2(a)). By contrast, in the ileum, despite a tendency for occludin protein expression to decrease in the $\mathrm{N}$ and $\mathrm{N}+\mathrm{TRP}$ groups, TRP supplementation seemed to have no influence (Fig. 2(b)). The concentrations of portal plasma LPS were almost 7-fold increased in the $\mathrm{N}$ group compared with that in the control group ( $P=0.005$; Fig. 2(c)). However, TRP supplementation led to no significant reduction in LPS concentrations in the N group (Fig. 2(c)).
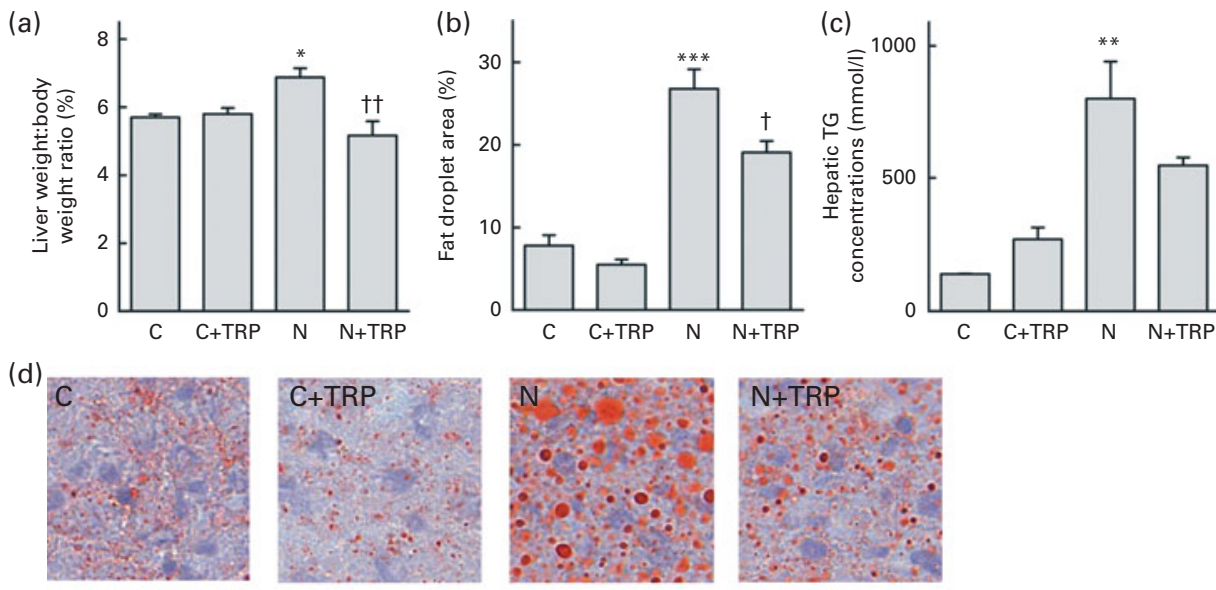

(e)
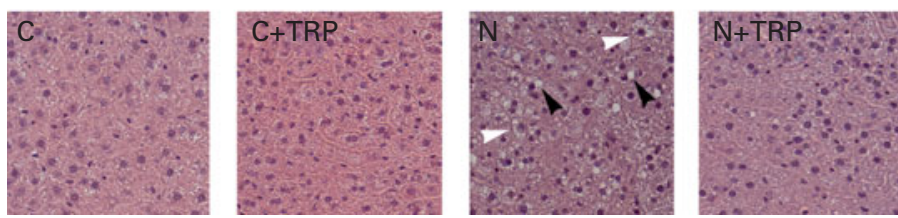

Fig. 1. Effect of tryptophan (TRP) supplementation on hepatic lipid accumulation. Liver weight:body weight ratio expressed in percentage (a), fat droplet area quantified by Oil Red O staining (b) and TAG concentrations (c). Values are means, with their standard errors represented by vertical bars $(n+4)$ ). C, control diet; C + TRP, control diet with TRP supplementation; N, non-alcoholic fatty liver disease (NAFLD) mice fed a fructose-rich diet; N + TRP, NAFLD mice fed a-fructose-rich diet with TRP supplementation. Mean value was significantly different from that of the $C$ group: ${ }^{*} P<0.05,{ }^{\star *} P<0.01,{ }^{* \star *} P<0.001$. Mean value was significantly different from that of the $N$ group: $\dagger P<0.05$, $\uparrow \dagger P<0.01$. Liver tissue sections were stained using Oil Red $O$ dye $(400 \times)(d)$ and haematoxylin and eosin $(200 \times)(e)$. Representative photomicrographs of the liver tissue sections are shown. Steatosis (black arrow heads) and cellular ballooning (white arrow heads) are marked for the $\mathrm{N}$ group. 

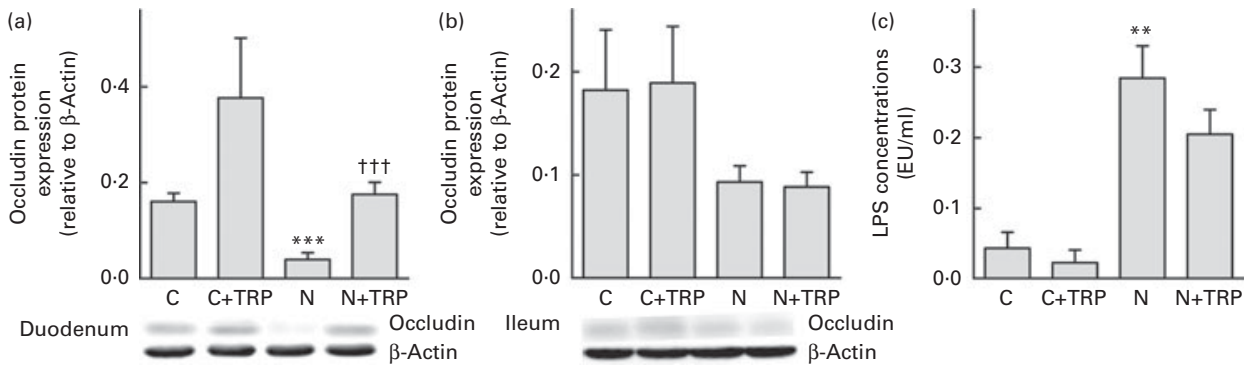

Fig. 2. Effect of tryptophan (TRP) supplementation on occludin protein expression in the duodenum. Occludin protein expression was quantified by Western blotting in the duodenum (a) and ileum (b). Representative blot images of occludin and $\beta$-actin as well as quantitative analysis results of the blots are shown $((a)$ and $(b))$. Lipopolysaccharide (LPS; endotoxin units $(E U) / m l)$ concentrations were measured in portal vein plasma (c). Values are means, with their standard errors represented by vertical bars $(n 4-6)$. C, control diet; C + TRP, control diet with TRP supplementation; N, non-alcoholic fatty liver disease (NAFLD) mice fed a fructose-rich diet; $N+$ TRP, NAFLD mice fed a fructose-rich diet with TRP supplementation. Mean value was significantly different from that of the $C$ group: ${ }^{\star \star} P<0.01,{ }^{\star \star \star} P<0.001$. ††† Mean value was significantly different from that of the $\mathrm{N}$ group $(P<0.001)$.

\section{Effect of tryptophan supplementation on the intestinal serotonergic system}

To determine whether measurable alterations in the intestinal serotonergic system were associated with the protective effect of TRP, SERT protein concentrations, TPH1 mRNA expression and 5-HT concentrations were quantified in the duodenum. Confirming our own previous data ${ }^{(7)}$, we observed a significant reduction in SERT protein concentrations by $56.4 \%$ in the $\mathrm{N}$ group compared with that in the control group $(P=0.002$; Fig. 3). TRP supplementation increased SERT protein $(P=0.02)$ expression in mice fed the fructose-rich diet (Fig. 3). As expected, TPH1 mRNA expression was increased $(P=0.01)$ in the $\mathrm{N}$ group, but it was not normalised by TRP supplementation in addition to fructose-rich diet feeding (data not shown). TRP supplementation had an influence neither on motility nor on overall 5-HT concentrations in the duodenum (Table 1).

\section{Discussion}

There is now growing experimental evidence that alterations in the intestinal barrier resulting in an increased translocation of bacterial products contribute to the development of $\operatorname{NAFLD}^{(7,27,28)}$. However, the triggers causing barrier impairment, LPS influx into the liver and hepatic lipid accumulation are largely unknown. We and others have validated that particular dietetic factors such as sugars, in particular, fructose ${ }^{(5)}$, and/or SFA ${ }^{(8,9)}$ play a role, but the underlying mechanisms have been described only rudimentarily.

Recent findings reported by our group have suggested that the intestinal serotonergic system, and especially SERT and 5-HT assessed in the present study, might be involved in the regulation of intestinal permeability and thus translocation of gut-derived bacterial $\operatorname{LPS}^{(7)}$. Such data raised the idea that the intestinal serotonergic system could be an interesting pharmacological target for the prevention of fatty liver disease.

Therefore, we chose the 5-HT precursor TRP as a dietary supplement, which has been investigated in various animal models ${ }^{(22,23)}$ and has no major side effects on liquid or food intake or on weight gain ${ }^{(29)}$. In the present study, we observed an increase in energy intake after TRP supplementation in the control mice but not in the NAFLD mice with TRP supplementation. The increase in energy intake did not have an effect on weight gain, which might be, among others, due to a slight but not significantly increased motility in the TRP-treated control mice.

In the present study, we clearly demonstrated the protective effect of TRP supplementation on the stabilisation of the intestinal barrier through the enhancement of occludin expression and the obvious reduction of fatty acid accumulation in the liver. The protective effect of TRP supplementation in our NAFLD model was not associated with an attenuation of intestinal translocation of bacterial LPS, possibly because of the partial normalisation of the tight junction impairment only in the duodenum but not in the ileum. Interestingly, L-TRP administration in the rat ileum has been found to reduce net $\mathrm{Na}^{+}$and fluid absorption, supposingly due to morphological changes ${ }^{(30)}$.

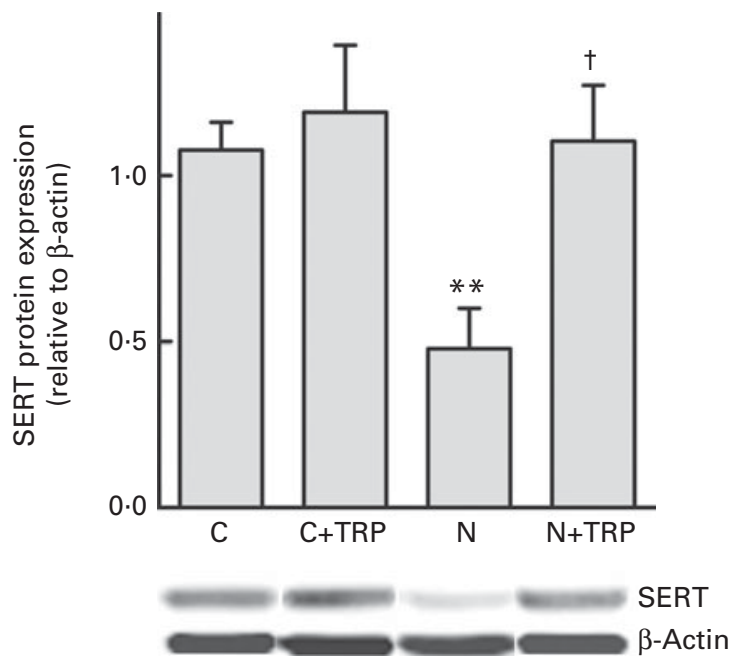

Fig. 3. Effect of tryptophan (TRP) supplementation on duodenal serotonin re-uptake transporter (SERT) protein expression. SERT protein expression in the duodenum was measured by Western blotting. Representative blot images of SERT and $\beta$-actin as well as the quantitative analysis results of the blots are shown. Values are means, with their standard errors represented by vertical bars ( $n 4-6)$. C, control diet; C + TRP, control diet with TRP supplementation; $N$, non-alcoholic fatty liver disease (NAFLD) mice fed a fructose-rich diet; N + TRP, NAFLD mice fed a fructose-rich diet with TRP supplementation. ${ }^{* *}$ Mean value was significantly different from that of the $C$ group $(P<0.01)$. † Mean value was significantly different from that of the $\mathrm{N}$ group $(P<0.05)$. 


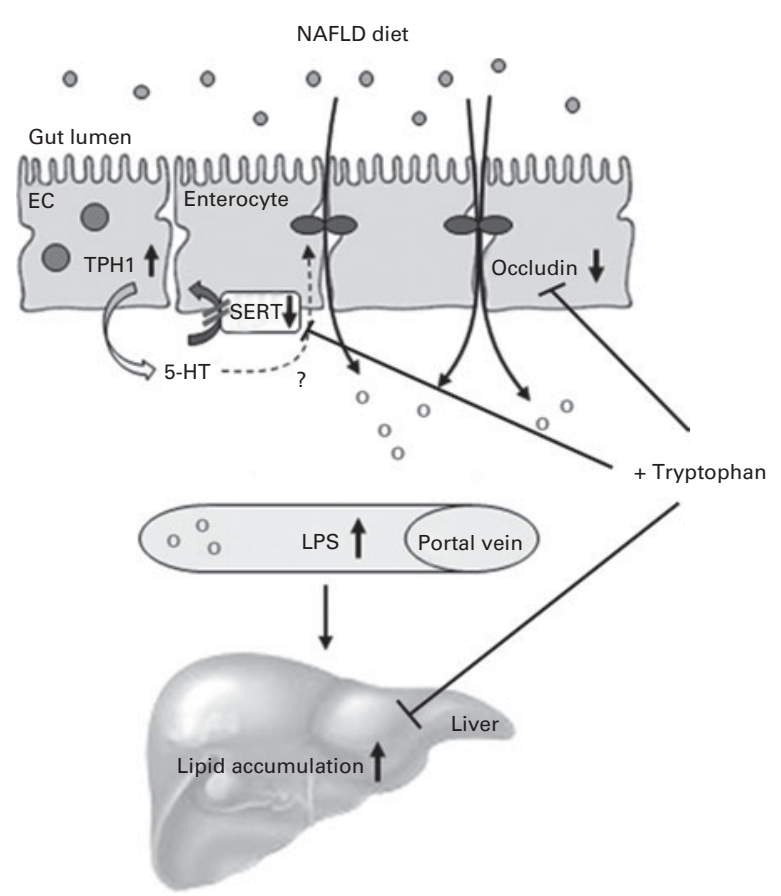

Fig. 4. Hypothesis on the possible influence of tryptophan (TRP) on nonalcoholic fatty liver disease (NAFLD). In our NAFLD model, we observed a decreased expression of serotonin (5-HT) re-uptake transporter (SERT) in the small intestine that might result in fortified signalling of bioavailable 5-HT in small intestinal tissue compared with that in the control group. Either via 5-HT or by other yet unknown mechanisms, the NAFLD-inducing fructoserich diet ( $\mathrm{N}$ group) used in the present study supposingly caused a decrease in occludin expression in the upper small intestine resulting in an impaired epithelial barrier and a consecutive increased translocation of bacteria $O$ or bacterial products such as lipopolysaccharides (LPS, O). In the $\mathrm{N}$ group, TRP supplementation normalised reduced SERT expression and assumingly improved barrier function in the upper intestine by enhancing occludin expression. Changes within the serotonergic system or the intestinal barrier might influence the reduction of hepatic lipid accumulation in our NAFLD model (effect of TRP supplementation (+Tryptophan) is shown with arrowheads; EC, enterochromaffin cells; TPH1, TRP hydroxylase 1.

Indeed, these ileal effects have been observed only at a concentration higher than $20 \mathrm{~mm}-\mathrm{L}-\mathrm{TRP}$, whereas lower concentrations have been found to rather stimulate net absorption ${ }^{(30)}$. As TRP was administered orally and not directly into the ileum in the present study, we observed no influence of TRP supplementation on intestinal barrier functions.

Earlier studies in rats have investigated the effect of TRP supplementation on fatty acid metabolism in the liver, confirming an increase in serum TAG concentrations but not in hepatic TAG concentrations after a single injection $(50 \mathrm{mg} / \mathrm{kg}$ body weight) or a treatment over $7 \mathrm{~d}(2.5 \mathrm{~g} / \mathrm{kg} \text { diet })^{(29,31)}$. In agreement with these findings, we found neither an increase nor a reduction in hepatic TAG concentrations or liver weight in the TRP-treated control mice. On the other hand, TRP supplementation reduced steatosis, cellular ballooning and lobular inflammation in the liver of the NAFLD mice. In close relation to our findings, a recent study has indicated positive effects of indoleamine 2,3-dioxygenase, an enzyme that mediates the catabolism of L-TRP to L-kynurenine, on liver inflammation and fibrosis ${ }^{(32)}$. In earlier studies,
TRP supplementation has been found to attenuate the development of NAFLD in laying hens ${ }^{(23,24)}$.

We not only found that TRP supplementation attenuated NAFLD but also found that it increased the lower intestinal expression of SERT in the $\mathrm{N}$ group. Lower intestinal expression of SERT in our NAFLD model was accompanied by liver steatosis, increased portal plasma LPS concentrations and tight-junction protein loss in the small intestine, as has been postulated here and previously ${ }^{(7)}$. Similarly, SERT knockout mice have been found to develop liver damage on being fed a glucose-rich diet, which induced no or minor damages in $\mathrm{C} 57 \mathrm{BL} / 6 \mathrm{~J}$ wild-type mice ${ }^{(7)}$. Our data indicated that fructose is more harmful than glucose in this respect, but in animals lacking SERT, glucose becomes equally harmful with regard to fatty liver disease as fructose in wild-type mice. Therefore, we suggest that SERT and thus the serotonergic system play a role in sugar-induced liver damage. Possibly, loss of SERT in the intestine leads to a dysregulation of 5-HT signalling that may evoke an enhanced inflammatory response as determined in IL-10 knockout and SERT/IL-10 double-knockout mice $^{(15)}$. The latter were characterised by high concentrations of TNF- $\alpha$, which modulates tight-junction proteins via myosin light-chain kinase activation ${ }^{(33)}$. Therefore, we assume that the induction of SERT might be an important mechanism, besides occludin enhancement, with regard to how TRP might attenuate NAFLD at the intestinal level (Fig. 4).

In contrast to previous findings reported by Ozer et al. ${ }^{(21,22)}$ no significant differences in small-intestinal 5-HT concentrations or motility were detected among the dietary groups. Ozer et $a l^{(21,22)}$ reported 5-HT concentrations and duodenal and ileal motility to be significantly increased in mice treated with TRP $(0 \cdot 1 \mathrm{~g} / \mathrm{kg}$ body weight per $24 \mathrm{~h}$, intraperitoneally, $7 \mathrm{~d})$, which might have resulted from the different doses and routes of administration (oral $v$. intraperitoneal). However, it is unclear whether overall concentrations of 5-HT in tissues are a reliable marker for 5-HT signalling. Therefore, we determined the mRNA concentration of TPH1, the rate-limiting enzyme of 5-HT synthesis, which was significantly increased on feeding the fructose-rich diet but not normalised by TRP supplementation. Nevertheless, we assume that TPH1 might be a more relevant marker for bioavailable 5-HT at the intercellular level than the overall 5-HT tissue concentration. Of course, other mechanisms such as central effects of TRP cannot be excluded and could contribute to the beneficial effects of TRP in the experimental NAFLD model used in the present study.

Our data provide the first evidence that oral supplementation of the amino acid TRP attenuates NAFLD in mice. In conclusion, we report that modulation of the upper intestinal barrier and the intestinal serotonergic system via TRP supplementation might be an important mechanism of protection from the development of NAFLD in mice. Adverse effects of TRP supplementation were not observed in our mouse model; however, these have to be considered when testing this approach in human subjects.

\section{Acknowledgements}

The present study was supported by grants from the 'Center for Nutritional Medicine' Tübingen/Hohenheim, Germany 
(TP7.AI to SCB), and the Competence Network of Obesity, group 'Obesity and GI tract', funded by the Federal Ministry of Education and Research (grant number 01GI0843 to SCB). The 'Center for Nutritional Medicine' Tübingen/Hohenheim and the Competence Network of Obesity had no role in the design and analysis of the study or in the writing of this article.

The authors' contributions are as follows: Y. R. and S. C. B. conceived and designed the experiments; Y. R., G. B., M. B. and A. H. carried out the experiments; Y. R., G. B., M. B. and A. H. analysed the data; Y. R. and S. C. B. interpreted the results of the experiments; Y. R. prepared the figures; Y. R. and A. H. drafted the manuscript; Y. R. and S. C. B. edited and revised the manuscript; Y. R., G. B., M. B., A. H. and S. C. B. approved the final version of the manuscript.

None of the authors has any conflicts of interest to declare.

\section{References}

1. Byrne CD (2012) Dorothy Hodgkin lecture 2012: nonalcoholic fatty liver disease, insulin resistance and ectopic fat: a new problem in diabetes management. Diabet Med 29, 1098-1107.

2. Adams KF, Schatzkin A, Harris TB, et al. (2006) Overweight, obesity, and mortality in a large prospective cohort of persons 50 to 71 years old. $N$ Engl J Med 355, 763-778.

3. Adams LA, Lymp JF, St Sauver J, et al. (2005) The natural history of nonalcoholic fatty liver disease: a populationbased cohort study. Gastroenterology 129, 113-121.

4. Spruss A \& Bergheim I (2009) Dietary fructose and intestinal barrier: potential risk factor in the pathogenesis of nonalcoholic fatty liver disease. J Nutr Biochem 20, 657-662.

5. Bergheim I, Weber S, Vos M, et al. (2008) Antibiotics protect against fructose-induced hepatic lipid accumulation in mice: role of endotoxin. J Hepatol 48, 983-992.

6. de Wit NJ, Afman LA, Mensink M, et al. (2012) Phenotyping the effect of diet on non-alcoholic fatty liver disease. J Hepatol 57, 1370-1373.

7. Haub S, Kanuri G, Volynets V, et al. (2010) Serotonin reuptake transporter (SERT) plays a critical role in the onset of fructose-induced hepatic steatosis in mice. Am J Physiol Gastrointest Liver Physiol 298, G335-G344.

8. Malhi H \& Gores GJ (2008) Molecular mechanisms of lipotoxicity in nonalcoholic fatty liver disease. Semin Liver Dis 28, 360-369.

9. Tetri LH, Basaranoglu M, Brunt EM, et al. (2008) Severe NAFLD with hepatic necroinflammatory changes in mice fed trans fats and a high-fructose corn syrup equivalent. Am J Physiol Gastrointest Liver Physiol 295, G987-G995.

10. Thuy S, Ladurner R, Volynets V, et al. (2008) Nonalcoholic fatty liver disease in humans is associated with increased plasma endotoxin and plasminogen activator inhibitor 1 concentrations and with fructose intake. J Nutr 138, 1452-1455.

11. Haub S, Ritze Y, Ladel I, et al. (2011) Serotonin receptor type 3 antagonists improve obesity-associated fatty liver disease in mice. J Pharmacol Exp Ther 339, 790-798.

12. Weber S, Volynets V, Kanuri G, et al. (2009) Treatment with the 5-HT3 antagonist tropisetron modulates glucose-induced obesity in mice. Int J Obes (Lond) 33, 1339-1347.

13. Wade PR, Chen J, Jaffe B, et al. (1996) Localization and function of a 5-HT transporter in crypt epithelia of the gastrointestinal tract. J Neurosci 16, 2352-2364.

14. Gershon MD (2004) Review Article: serotonin receptors and transporters - roles in normal and abnormal gastrointestinal motility. Aliment Pharmacol Ther 20, Suppl. 7, 3-14.
15. Haub S, Ritze Y, Bergheim I, et al. (2010) Enhancement of intestinal inflammation in mice lacking interleukin 10 by deletion of the serotonin reuptake transporter. Neurogastroenterol Motil 22, 826-834, e229.

16. Bischoff SC, Mailer R, Pabst O, et al. (2009) Role of serotonin in intestinal inflammation: knockout of serotonin reuptake transporter exacerbates 2,4,6-trinitrobenzene sulfonic acid colitis in mice. Am J Physiol Gastrointest Liver Physiol 296, G685-G695.

17. Nylander O \& Pihl L (2006) Luminal hypotonicity increases duodenal mucosal permeability by a mechanism involving 5-hydroxytryptamine. Acta Physiol (Oxf) 186, 45-58.

18. Stull MA, Pai V, Vomachka AJ, et al. (2007) Mammary gland homeostasis employs serotonergic regulation of epithelial tight junctions. Proc Natl Acad Sci U S A 104, 16708-16713.

19. Yamada T, Inui A, Hayashi N, et al. (2003) Serotonin stimulates endotoxin translocation via 5-HT3 receptors in the rat ileum. Am J Physiol Gastrointest Liver Physiol 284, G782-G788.

20. Noguchi T, Nishino M \& Kido R (1973) Tryptophan 5-hydroxylase in rat intestine. Biochem J 131, 375-380.

21. Ozer C, Gonul B, Ercan ZS, et al. (2007) The effect of tryptophan administration on ileum contractility and oxidant status in mice. Amino Acids 32, 453-458.

22. Ozer C, Gonul B, Take G, et al. (2004) Tryptophan administration increase contractility and change the ultrastructure of mice duodenum. Amino Acids 27, 215-220.

23. Akiba Y, Takahashi K, Horiguchi M, et al. (1992) L-Tryptophan alleviates fatty liver and modifies hepatic microsomal mixed function oxidase in laying hens. Comp Biochem Physiol Comp Physiol 102, 769-774.

24. Rogers SR \& Pesti GM (1992) Effects of tryptophan supplementation to a maize-based diet on lipid metabolism in laying hens. Br Poult Sci 33, 195-200.

25. Lesurtel M, Soll C, Graf R, et al. (2008) Role of serotonin in the hepato-gastrointestinal tract: an old molecule for new perspectives. Cell Mol Life Sci 65, 940-952.

26. Kleiner DE, Brunt EM, Van Natta M, et al. (2005) Design and validation of a histological scoring system for nonalcoholic fatty liver disease. Hepatology 41, 1313-1321.

27. Brun P, Castagliuolo I, Di Leo V, et al. (2007) Increased intestinal permeability in obese mice: new evidence in the pathogenesis of nonalcoholic steatohepatitis. Am J Physiol Gastrointest Liver Physiol 292, G518-G525.

28. Cani PD, Bibiloni R, Knauf C, et al. (2008) Changes in gut microbiota control metabolic endotoxemia-induced inflammation in high-fat diet-induced obesity and diabetes in mice. Diabetes 57, 1470-1481.

29. Fears R \& Murrell EA (1980) Tryptophan and the control of triglyceride and carbohydrate metabolism in the rat. $\mathrm{BrJ}$ Nutr 43, 349-356.

30. Teichberg S, Wapnir RA, Zdanowicz M, et al. (1989) Morphologic and functional alterations in absorptive epithelial cells during L-tryptophan induced inhibition of net sodium and fluid absorption in the rat ileum. Lab Invest 60, 88-101.

31. Sakurai T, Miyazawa S, Shindo Y, et al. (1974) The effect of tryptophan administration on fatty acid synthesis in the liver of the fasted normal rat. Biochim Biophys Acta 360 , $275-288$

32. Nagano J, Shimizu M, Hara T, et al. (2013) Effects of indoleamine 2,3-dioxygenase deficiency on high-fat diet-induced hepatic inflammation. PLoS One 8, e73404.

33. Turner JR (2009) Intestinal mucosal barrier function in health and disease. Nat Rev Immunol 9, 799-809. 